\title{
The Rare Diseases Clinical Research Network's Organization and Approach to Observational Research and Health Outcomes Research
}

\author{
Jeffrey P. Krischer, Ph.D. ${ }^{1}$, Rashmi Gopal-Srivastava, Ph.D. ${ }^{2}$, Stephen. C. Groft, Pharm.D. ${ }^{2}$, and \\ David J. Eckstein, Ph.D. ${ }^{2}$ for the Rare Diseases Clinical Research Network
}

'University of South Florida, Tampa, FL, USA; ${ }^{2}$ Office of Rare Diseases Research, National Center for Advancing Translational Sciences

(NCATS), Bethesda, MD, USA.

Established in 2003 by the Office of Rare Diseases Research (ORDR), in collaboration with several National Institutes of Health (NIH) Institutes/Centers, the Rare Diseases Clinical Research Network (RDCRN) consists of multiple clinical consortia conducting research in more than 200 rare diseases. The RDCRN supports longitudinal or natural history, pilot, Phase I, II, and III, case-control, cross-sectional, chart review, physician survey, bio-repository, and RDCRN Contact Registry (CR) studies. To date, there have been 24,684 participants enrolled on 120 studies from 446 sites worldwide. An additional 11,533 individuals participate in the CR. Through a central data management and coordinating center (DMCC), the RDCRN's platform for the conduct of observational research encompasses electronic case report forms, federated databases, and an online CR for epidemiological and survey research. An ORDRgoverned data repository (through dbGaP, a database for genotype and phenotype information from the National Library of Medicine) has been created. DMCC coordinates with ORDR to register and upload study data to dbGaP for data sharing with the scientific community. The platform provided by the RDCRN DMCC has supported 128 studies, six of which were successfully conducted through the online CR, with 2,352 individuals accrued and a median enrollment time of just 2 months. The RDCRN has built a powerful suite of web-based tools that provide for integration of federated and online database support that can accommodate a large number of rare diseases on a global scale. RDCRN studies have made important advances in the diagnosis and treatment of rare diseases.

KEY WORDS: rare diseases network; multi-institutional clinical research; outcomes; patient advocacy groups.

J Gen Intern Med 29(Suppl 3):S739-44

Electronic supplementary material The online version of this article (doi:10.1007/s11606-014-2894-x) contains supplementary material, which is available to authorized users.

Published online July 17, 2014
DOI: $10.1007 / \mathrm{s} 11606-014-2894-\mathrm{x}$

(c) Society of General Internal Medicine 2014

\section{INTRODUCTION}

The Rare Diseases Clinical Research Network (RDCRN), established in 2003 by the Office of Rare Diseases Research (ORDR) at the National Institutes of Health (NIH), currently located in the National Center for Advancing Translational Sciences (NCATS), comprises 17 consortia (each studying three or more rare diseases, Fig. 1), the DMCC, and more than 90 patient advocacy groups (PAGs). ${ }^{1-5}$ (On-line Appendix) The ORDR/NCATS leads the funding and coordination of this trans-NIH program. Each consortium receives $1.25 \mathrm{M}$ total cost/year, and in the current grant cycle, NIH spends approximately \$25 million/ year (half provided by ORDR/NCATS). The purpose of the RDCRN is to facilitate clinical research in rare diseases through 1) longitudinal studies of individuals with rare diseases, clinical studies and/or phase I, II and II/III trials; 2) training of clinical investigators in rare diseases research; and 3) pilot and demonstration projects. It is also serves as a test bed for distributed clinical data management that incorporates novel approaches and technologies for data management, data mining, and data sharing across rare diseases, data types, and platforms; and to facilitate access to information related to rare diseases to basic and clinical researchers, academic and practicing physicians, patients, and the lay public. Project scientists from eight NIH Institutes and Centers are actively involved as collaborators.

The purpose of this paper is to describe the organization and structure of the RDCRN, its accomplishments, and the opportunities and challenges it faces as it enters its second decade. Among its goals are the training of new investigators in rare diseases research and improving access to information related to rare diseases for basic and clinical researchers, academic and practicing physicians, patients, and the lay public. This paper describes the progress to date 
Rare Diseases Clinical Research Network (RDCRN)

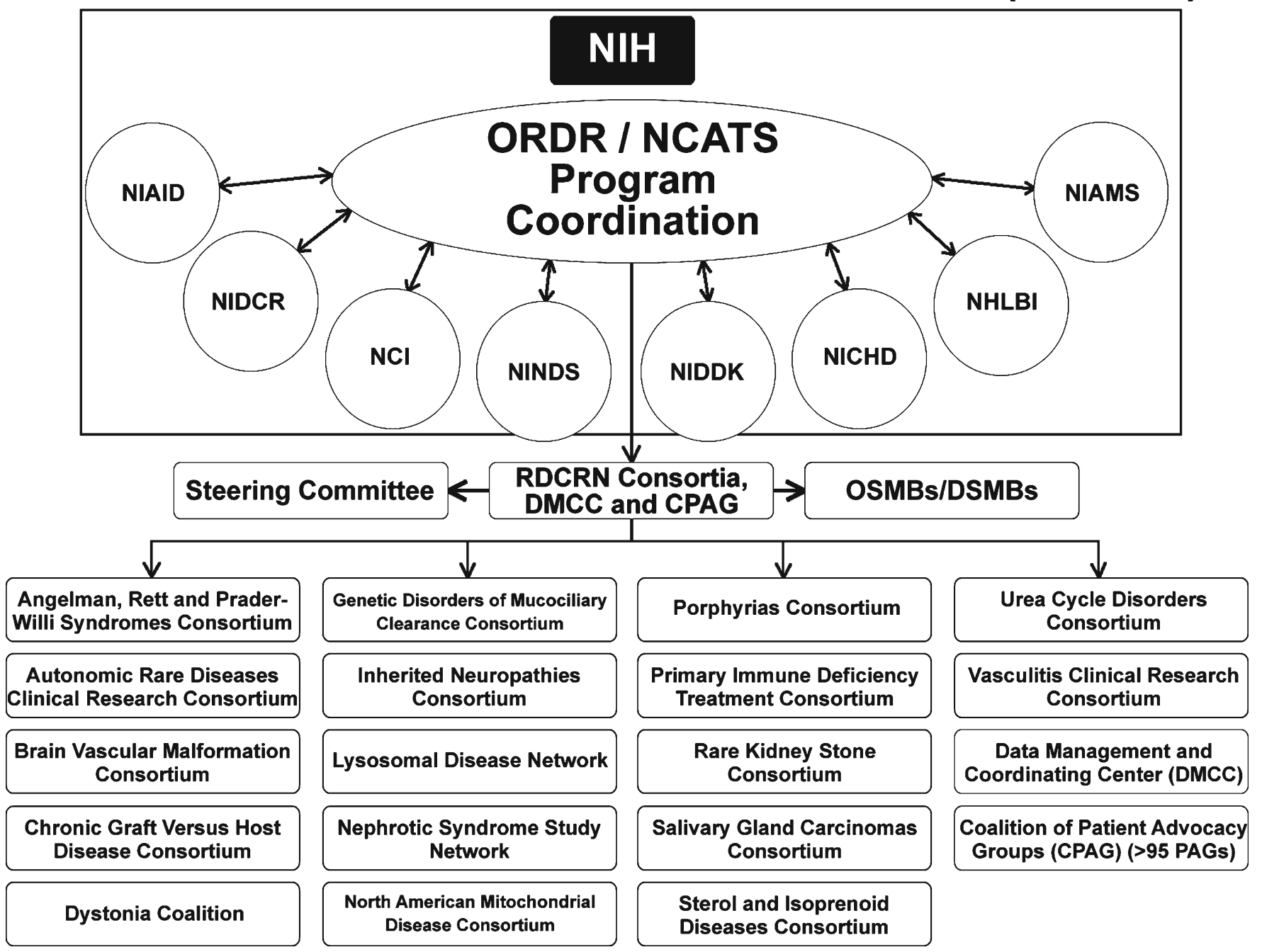

Figure 1.. The Consortia of the Rare Diseases Clinical Research Network. *National Institutes of Health (NIH), Office of Rare Diseases Research/National Center for Advancing Translational Sciences (ORDR/NCATS), National Institute of Allergy and Infectious Diseases

(NIAID), National Institute of Dental and Craniofacial Research (NIDCR), National Cancer Institute (NCI), National Institute of Neurological Disorders and Stroke (NINDS), National Institute of Diabetes and Digestive and Kidney Diseases (NIDDK), National Institute of Child Health and Human Development (NICHD), National Heart, Lung and Blood Institute (NHLBI), National Institute of Arthritis and Musculoskeletal and Skin Diseases (NIAMS), Observational Study Monitoring Board/Data and Safety Monitoring Board (OSMB/DSMB).

in terms of treatment outcomes as well as the resource that has been created for future studies.

\section{RDCRN ORGANIZATION AND STRUCTURE}

The RDCRN is a network of consortia, each focusing on a different group of rare diseases. Consortia that are part of the RDCRN were selected by a peer-review process in response to a funding opportunity announcement from the ORDR in 2003 and again in 2009. Program coordination for RDCRN is provided by ORDR/NCATS. Each consortium is also managed by the appropriate NIH Institute/ Center, depending upon its disease focus. That cooperative agreement award provides funds to perform collaborative multi-site clinical research in rare diseases, train new investigators in rare diseases research, and provide content for an internet resource site on rare diseases. Each consortium consists of clinical investigators, institutions, and relevant organizations, including the integration of PAGs into its research program. Since rare diseases are diverse, the nature of feasible clinical research varies, and the individual consortia are responsible for the design and implementation of their own clinical studies. The DMCC provides data management and support, integrating protocols, forms, and research tools to the RDCRN.

Study development begins within the individual consortium and follows a template developed by the DMCC. This facilitates the process, in that the essential elements of a study protocol and the informed consent/assent forms are immediately available, along with approved language for 
standard protocol content (e.g., adverse event reporting) and processes for transfer of data to a common RDCRN database maintained by the DMCC. Each protocol is reviewed either by one of several standing Data Safety and Monitoring Boards (DSMBs) or by a process internal to the NIH Institute/Center prior to study activation. The DSMBs also provide ongoing review of open studies. All sites enrolling on RDCRN protocols are visited by the DMCC according to RDCRN standards.

Consortia with their own data-collection capability can enroll participants and collect study data using those systems. Alternatively, the web-based tools developed by the DMCC are available for enrollment, data collection (i.e., electronic case report forms, direct data entry for patientreported outcomes, or automated transfers from laboratories or images from radiology departments), and cumulative reporting of accrual rates, protocol compliance, and data completeness. The DMCC systems also include methods for reporting and review of adverse events by an assigned medical monitor for each consortium, a pharmacy system to track drug distribution and re-stocking of investigational agents, and a biospecimen tracking system to follow samples collected in the clinic to a consortium reference laboratory and to link laboratory results with the clinical data collected. ${ }^{6}$

The DMCC website also contains public-facing pages offering information on each consortium, current studies, and the RDCRN patient Contact Registry (CR). ${ }^{7,8}$ Information provided by the consortium may include practice guidelines for rare diseases, training opportunities, and links to PAGs. Current study information provides a synopsis of each study, lists sites where it is currently open for enrollment, and offers contact information for patients and health care providers.

The website has information on the more than 200 diseases studied by the RDCRN. In addition, it houses or directs visitors to other vital information, such as a complete listing of all open protocols, history and background information on the RDCRN and its funding institutions, and access to web sites such as the Spotlight on Rare Diseases e-newsletter, the Conference on Clinical Research for Rare Diseases (CCRRD); the Coalition of Patient Advocacy Groups, which consists of all PAGs participating in the RDCRN as research partners with individual consortia; and the RDCRN Consortium Members website application. Each RDCRN consortium has its own public website, hosted on the RDCRN website. The RDCRN consortium web pages contain key information such as diseases being studied, contact information for the consortia investigators and supporting staff, accruing protocols, and participating PAGs.

The members' website is an internal secure web-site that is password protected. Through it, all members access administrative RDCRN information, protocol documents, training materials, RDCRN study monitoring reports, and committee calendars, as well as subject data such as electronic case report forms, biospecimen tracking, and pharmacy data. The main members web page includes a patient accrual table that details real-time accrual across all consortia, participating sites, and studies. Individualized consortium-specific internal resource pages and document management areas are available for all RDCRN consortia. The consortium-level resource web pages can be used to post and share meeting information, protocol documents, data, and reports. Additionally, the consortia can post events, meetings, or calls to the consortium calendar, add and maintain consortium-specific folders, and email other members of the consortium or user-defined subscriber/ group lists through the resource pages. The design and structure of the RDCRN members web site allows it to be easily scalable and expandable according to the needs of the RDCRN. The infrastructure has been developed so that as consortia are added or needs of the consortia expand, modular components of the web site can be expanded as well. Each system can be further customized to adhere to a specific protocol or consortium requirements.

The patient $\mathrm{CR}$ is a HIPAA-compliant online system designed by the DMCC to collect contact and diagnosis information volunteered by rare-disease patients interested in clinical research participation. Individuals registering on the website will receive disease-specific or consortiumspecific notifications from the RDCRN when studies open or opportunities for participating in research become available. The automated communication system for the RDCRN CR is database driven, making it highly efficient. The protocol information (e.g., study description, eligibility criteria, etc.) that is sent to registrants is automatically extracted from the DMCC protocol management database and inserted into standardized email or letter templates. The system monitors the addition of new protocols or changes in information (e.g., study sites opening or closing) from internal DMCC databases and automatically notifies the contact registrants of such changes as they occur. The primary focus of the $\mathrm{CR}$ is to establish two-way communication about the activities of the RDCRN. It is not a patient registry. Over the last 2 years, the RDCRN CR has provided survey access to research population that extends far beyond patients seen at the consortium clinical sites. ${ }^{9}$

\section{RDCRN OUTCOMES}

During the first 5 years of the RDCRN (2003-2008), 40 studies were opened to accrual by ten consortia with a median time from protocol concept initiation to activation of 2.1 years. During the second 5 years of the RDCRN (2009-2013), 88 studies were opened by 17 consortia, and the median protocol development time was reduced to 0.7 years. Patient enrollment grew from 5,633 to 24,684 . Of these recently opened 88 studies, 52 (59\%) are longitudinal 
or natural history (observational) studies; nine (10\%) are pilot studies; two (2\%), three (4\%), and four (5\%) are Phase I, II, and III studies, respectively; one (1\%) is a casecontrol study, one (1\%) is a cross-sectional study, and two (2\%) each are chart reviews, physician surveys, or biorepository studies. Six studies have been conducted using the CR, accruing 2,406 registrants. Notably, these CR studies met their accrual targets within 2 to 4 months of opening (Table 1).

Over 14,776 individuals from over 90 countries have subscribed to the RDCRN CR, which was designed for access by the general public beyond those enrolled in RDCRN studies at RDCRN sites. Over 142,000 email notices have been sent to registrants since 1 August 12009 to notify them of new studies, new sites, online protocols, and as part of web-based survey research protocols.

Over the last 5 years, the RDCRN has provided training opportunities for 158 trainees, published 510 journal articles, 62 books and book chapters, and made 122 conference presentations. While the body of research results continues to add to our knowledge in many rare diseases, it is useful to highlight a few projects that demonstrate the efficacy of new treatments or diagnostic advances for rare diseases that have made a significant impact on the lives of those affected.

The first example of the productivity of the RDCRN is the Multicenter International Lymphangioleiomyomatosis (LAM) Trial of the Efficacy and Safety of Sirolimus (MILES) ${ }^{10}$ LAM is a cystic lung disease of women, associated with mutations in tuberous sclerosis complex (TSC) genes that control mTOR signaling. Sirolimus suppresses increased mTOR activity observed with TSC inactivation.

The Rare Lung Disease Consortium conducted a 24month controlled trial of sirolimus in patients with LAM and moderate lung impairment. Coordinated by the DMCC, this study involved international sites, central laboratory determinations of sirolimus blood levels and dose modifications to maintain therapeutic windows, all while maintaining the double-blinded design. Change in functional residual lung capacity and measures of quality of life and functional performance differed between groups during the treatment year, favoring sirolimus. The study concluded that on 1 year of oral sirolimus, patients with LAM and moderate respiratory impairment experienced significant but modest improvements in lung function and measures of quality of life or functional performance. Efforts are continuing to obtain an indication for sirolimus in LAM, which would be the first approved treatment for this disease.

Each consortium in the RDCRN is required to have a pilot study program. One example was the first clinical trial of the use of losartan, an antifibrotic agent, to treat idiopathic pulmonary fibrosis (IPF). ${ }^{11}$ IPF is a progressive interstitial lung disease for which there are no effective therapies. The primary objective was to evaluate the effect of losartan on progression of IPF, measured by the change in percentage of predicted forced vital capacity (\%FVC) after 12 months. Twelve of 17 patients with IPF had stable or improved \%FVC at study month 12 . The study concluded that over 12 months Losartan stabilized lung function in patients with IPF and that Losartan is a promising agent for the treatment of IPF to be explored further in a Phase II study.

A third example is nondystrophic myotonias (NDMs), which are rare diseases caused by mutations in skeletal muscle ion channels. ${ }^{12}$ Patients experience delayed muscle relaxation, causing functionally limiting stiffness and pain. Mexiletine-induced sodium channel blockade reduced myotonia in small studies; however, as is common in rare diseases, larger studies of safety and efficacy had not been considered feasible. However, the multi-institutional RDCRN made it possible to undertake a randomized, double-blind, placebo-controlled, two-period crossover study at seven neuromuscular referral centers in four countries. ${ }^{9}$ This study successfully employed an interactive voice-response system as a daily diary of patient-reported outcomes. In this RDCRN study of patients with NDMs, 4 weeks of mexiletine resulted in improved patient-reported stiffness compared with placebo.

Some RDCRN studies sought to evaluate promising diagnostic methods and others to establish the natural history of a rare disease or a biomarker for associated health risks.

Table 1. Rare Diseases Clinical Research Network Contact Registry Studies

\begin{tabular}{|c|c|c|}
\hline Consortium & Title & Accrual \\
\hline Vasculitis Clinical Research Consortium & Reproductive Health of Men and Women with Vasculitis & $\begin{array}{l}N=467 \\
\text { Accrual goal met in } 2 \text { months. }\end{array}$ \\
\hline Vasculitis Clinical Research Consortium & $\begin{array}{l}\text { Illness Perceptions, Fatigue, and Function in Systemic } \\
\text { Vasculitis (The VCRC Vasculitis Perception (VIP) Study) }\end{array}$ & $\begin{array}{l}N=707 \\
\text { Accrual goal met in } 2 \text { months. }\end{array}$ \\
\hline Inherited Neuropathies Consortium & $\begin{array}{l}\text { Development and Validation of a Disability Severity Index } \\
\text { for Charcot-Marie-Tooth Disease (CMT) }\end{array}$ & $\begin{array}{l}N=249 \\
\text { Accrual goal met in } 4 \text { months. }\end{array}$ \\
\hline Vasculitis Clinical Research Consortium & $\begin{array}{l}\text { Educational Needs of Patients with Systemic Vasculitis- } \\
\text { An International Study }\end{array}$ & $\begin{array}{l}N=386 \\
\text { Accrual goal met in } 2 \text { months. }\end{array}$ \\
\hline Nephrotic Syndrome Study Network & $\begin{array}{l}\text { Assessment of Educational Experience for Patients with } \\
\text { Newly Diagnosed Nephrotic Syndrome }\end{array}$ & $\begin{array}{l}N=186 \\
\text { Accrual goal met in } 4 \text { months. }\end{array}$ \\
\hline Inherited Neuropathies Consortium & $\begin{array}{l}\text { An Analysis of the Symptomatic Domains Most Relevant to } \\
\text { Charcot Marie Tooth Neuropathy (CMT) Patients }\end{array}$ & $N=411$ Recruited in 2 months. \\
\hline
\end{tabular}


For example, several studies suggest that nasal nitric oxide (nNO) measurement could be a test for primary ciliary dyskinesia (PCD), but the procedure and interpretation have not been standardized. The Genetic Disorders of Mucociliary Clearance Consortium undertook an assessment of a standard protocol for measuring $\mathrm{nNO}$ to establish a disease-specific cut-off value at one site, then validate at six other sites. ${ }^{13}$ At the lead site, $\mathrm{nNO}$ was prospectively measured in individuals later confirmed to have PCD by ciliary ultrastructural defects $(n=143)$ or DNAH11 mutations $(n=6)$; along with 78 healthy controls and 146 controls with diseases including asthma $(n=37)$, cystic fibrosis (CF) $(n=77)$, and chronic obstructive pulmonary disease (COPD) $(n=32)$. In collaboration with the DMCC, a disease-specific cut-off value was determined using generalized estimating equations. This cut-off identified 70 of the $71(98.6 \%)$ participants with confirmed PCD. The study concluded that using a standardized protocol in multi-center studies, nasal NO measurement accurately identifies individuals with $\mathrm{PCD}$, supporting its utility as a test contributing to the clinical diagnosis of PCD.

\section{DISCUSSION}

The pace of research in rare diseases has been accelerated by the RDCRN. The median time from study concept to initiation has shortened, attesting to the streamlining of the process, the availability of key infrastructure, and the dedication of the partnership among investigators, PAG members, and the NIH. Protocol development time compares quite favorably to the results reported by the National Cancer Institute on cooperative group protocol development, which has been under way for a much longer time. ${ }^{14}$

Establishing the patient $\mathrm{CR}$ has become a major initiative of the RDCRN. Connecting with patients online is a novel and important way to reach patients beyond the referral catchment of major academic centers. Increased access to participants in ongoing research promotes equality of access and representativeness in medical research, provides opportunities for patients to interact with disease experts, and increases cohort sample sizes. This is particularly important for rare diseases, where access to experts can be limited and study sample sizes tend to be small. The opportunity to promote education and awareness about research in rare diseases expands the potential research community, and is important for both study recruitment and subject retention. ${ }^{15}$ The RDCRN includes several proven examples of its successful utilization of the CR to collect data directly from patients to conduct novel research studies that address questions of importance to patients. Features of the $\mathrm{CR}$ that make it such a useful tool are 1) only the DMCC IRB approval is needed, since the DMCC's university is the host of the website, obviating the need to obtain multiple IRB approvals, as is necessary in multi-institutional studies; 2) the DMCC has HIPAA approval to collect patient identifiers and contact information permitting two-way communication; 3) an IRB/HIPAA-approved feature is the ability to share information with RDCRN investigators (and more recently with PAGs) at the discretion of the registrant, facilitating the linkage between patient and investigator to boost protocol enrollment.

The data from all of the studies conducted by the RDCRN consortia have been collected in a database maintained by the DMCC, making it a valuable resource for future research. International data standards and recommended common data elements are used to provide a basis for comparison with other studies. ${ }^{16-18}$ Numerous RDCRN studies have related clinical observations to patient quality-of-life outcomes, ${ }^{19-27}$ and reflect a growing interest in incorporating patient-reported outcomes in both clinical trials and natural history studies. By every measure, the RDCRN continues to be a successful model for rare-diseases research. ${ }^{28}$

The RDCRN response to the challenges of conducting health outcomes research in rare diseases has been to create multi-institutional consortia, including sites in 14 countries, focusing on specific diseases, and employing technologies that are scalable, extensible, and generalizable to provide a common set of tools to facilitate research through the DMCC. Those tools permit a wide range of study designs, safety monitoring, biospecimen tracking, research pharmacy drug inventories, image collection, mobile and interactive voice technologies, and extensive study monitoring and analysis. The embodiment of common data elements and standards in the RDCRN enables the exchange of information across studies and across diseases, enhancing the value of the accumulating data to the larger scientific community.

The CR permits ascertainment and comparison of practice patterns and outcomes achieved within the consortium centers of excellence and the broader community at large. While the CR has proven to be an efficient and useful tool, it is not without its limitations. Because it is ascertained directly from patients and their families, the information that can reliably be collected may be limited or at least subject to interpretation. While it can be argued that those affected by rare diseases become expert in their disease and its outcomes, research that depends directly upon patient reporting needs to be carefully designed.

With this caution in mind, the desire to integrate patientreported outcomes into the conduct of clinical trials makes it imperative to develop instruments applicable to these disease settings and to find efficient ways to administer them. The challenge for the future is to continue to adapt these technologies to enhance research in rare diseases.

Acknowledgements: The RDCRN Steering Committee is aware of and has approved publication in this journal. The contents are solely the responsibility of the authors and do not necessarily 
represent the official views of NINDS or ORDR. The views expressed in written materials or publications do not necessarily reflect the official policies of the Department of Health and Human Services.

Funding: The RDCRN is funded and supported by the Office of Rare Diseases Research, National Center of Advancing Translational Sciences, in collaboration with the National Institute of Child Health and Human Development (NICHD), the National Institute of Arthritis and Musculoskeletal and Skin Diseases (NIAMS), the National Institute of Neurological Disorders and Stroke (NINDS), the National Institute of Diabetes and Digestive and Kidney Diseases (NIDDK), the National Institute of Allergy and Infectious Diseases (NIAID), the National Cancer Institute (NCI), the National Heart, Lung and Blood Institute (NHLBI), and the National Institute of Dental and Craniofacial Research (NIDCR).

Conflicts of Interest: The authors declare that they do not have a conflict of interest.

Corresponding Author: Jeffrey P. Krischer, Ph.D.; 3650 Spectrum Blvd, Tampa, FL 33612, USA (e-mail: jpkrischer@epi.usf.edu).

\section{REFERENCES}

1. Health Promotion and Disease Prevention Amendments of 1984, Pub. L. No. 98-551, 98 Stat. 2815 (Oct. 30, 1984).

2. Hampton T. Rare disease research gets boost. JAMA. 2006;295(24):2836-2838.

3. Akers A, Ball KL, Clancy $\mathbf{M}$, et al. the Brain Vascular Malformation Consortium. Brain Vascular Malformation Consortium: overview, progress, and future directions. J Rare Disorders. 2013;1(1):1-15.

4. Chronic Graft Versus Host Disease Consortium. Rationale and design of the chronic GVHD cohort study: improving outcomes assessment in chronic GVHD. Biol Blood Marrow Transplant. 2011;17(8):1114-1120.

5. Gadegbeku CA, Gipson DS, Holzman LB, et al. Design of the Nephrotic Syndrome Study Network (NEPTUNE) to evaluate primary glomerular nephropathy by a multidisciplinary approach. Kidney Int. 2013;83(4):749-756.

6. Richesson RL, Malloy JF, Paulus K, Cuthbertson D, Krischer JP. An automated standardized system for managing adverse events in clinical research networks. Drug Saf. 2008;31(10):807-822

7. Richesson RL, Lee HS, Cuthbertson D, Lloyd J, Young K, Krischer JP. An automated communications system in a contact registry for persons with rare diseases: scalable tools for identifying and recruiting clinical research participants. Contemp Clin Trials. 2009;30(1):55-62

8. Richesson RL, Sutphen R, Shereff D, Krischer JP. The rare diseases clinical research network contact registry update: features and functionality. Contemp Clin Trials. 2012;33(4):647-656.

9. Clowse ME, Richeson RL, Pieper C, Merkel PA. for the Vasculitis Clinical Research Consortium. Pregnancy outcomes among patients with vasculitis. Arthritis Care Res. 2013;65(8):1370-1374.

10. McCormack FX, Inoue $\mathbf{Y}$, Moss $\mathbf{J}$, Singer LG, et al. the National Institutes of Health Rare Lung Diseases Consortium; the MILES Trial
Group. Efficacy and safety of sirolimus in lymphangioleiomyomatosis. N Engl J Med. 2011;364(17):1595-1606.

11. Couluris M, Kinder BW, Xu P, Gross-King M, Krischer J, Panos RJ. Treatment of idiopathic pulmonary fibrosis with losartan: a pilot project. Lung. 2012;190(5):523-527.

12. Statland JM, Bundy BN, Wang Y, et al. Mexiletine for symptoms and signs of myotonia in nondystrophic myotonia: a randomized controlled trial. JAMA. 2012;308(13):1357-1365.

13. Knowles MR, Daniels LA, Davis SD, Zariwala MA, Leigh Mw. Primary ciliary dyskinesia. Recent advances in diagnostics, genetics, and characterization of clinical disease. Am J Resp. Crit Care Med. 2013;188(8):913-922.

14. Abrams JS, Mooney MM, Zwiebel JA, et al. Implementation of timeline reforms speeds initiation of National Cancer Institute-sponsored trials. JNCI. 2013;105(13):954-959.

15. Griggs RC, Batshaw M, Dunkle M, et al. Clinical research for rare disease: opportunities, challenges, and solutions. Mol Genet Metab. 2009;96(1):20-26.

16. Richesson R, Syed A, Guillette H, Tuttle MS, Krischer J. A web-based SNOMED CT browser: distributed and real-time use of SNOMED CT during the clinical research process. Stud Health Technol Inform. 2007; 129(Pt 1):631-635.

17. Richesson RL, Smith SB, Malloy J, Krischer JP. Achieving standardized medication data in clinical research studies: two approaches and applications for implementing RxNorm. J Med Syst. 2010;34(4):651-657.

18. Richesson RL, Nadkarni P. Data standards for clinical research data collection forms: current status and challenges. J Am Med Inform Assoc. 2011;18(3):341-346.

19. Pidala J, Kurland B, Chai $\mathbf{x}$, et al. Patient-reported quality of life is associated with severity of chronic graft-versus-host disease as measured by NIH criteria: report on baseline data from the Chronic GVHD Consortium. Blood. 2011;117(17):4651-4657.

20. Pidala J, Kurland BF, Chai X, et al. Sensitivity of changes in chronic graft-versus-host disease activity to changes in patient-reported quality of life: results from the Chronic Graft-versus-Host Disease Consortium. Haematologica. 2011;96(10):1528-1535.

21. Burns J, Ramchandren S, Ryan MM, et al. Determinants of reduced health-related quality of life in pediatric inherited neuropathies. Neurology. 2010;75(8):726-731.

22. Burns J, Ryan MM, Ouvrier RA. Quality of life in children with CharcotMarie-Tooth disease. J Child Neurol. 2010;25(3):343-347.

23. Walsh M, Mukhtyar C, Mahr A, et al. Health-related quality of life in patients with newly diagnosed antineutrophil cytoplasmic antibodyassociated vasculitis. Arthritis Care Res. 2011;63(7):1055-1061.

24. Tomasson G, Boers M, Walsh M, et al. Assessment of health-related quality of life as an outcome measure in granulomatosis with polyangiitis (Wegener's). Arthritis Care Res. 2012;64(2):273-279.

25. Lane JB, Lee HS, Smith LW, et al. Clinical severity and quality of life in children and adolescents with Rett syndrome. Neurology. 2011;77(20):1812-1818.

26. Herlyn K, Hellmich B, Seo P, Merkel PA. Patient-reported outcome assessment in vasculitis may provide important data and a unique perspective. Arthritis Care Res. 2010;62(11):1639-1645.

27. Reilly MM, Shy ME, Muntoni F, Pareyson D. 168th ENMC International Workshop: outcome measures and clinical trials in Charcot-MarieTooth disease (CMT). Neuromuscul Disord. 2010;20(12):839-846

28. Groft SC, Gopal-Srivastava R. A model for collaborative clinical research in rare diseases: experience from the Rare Disease Clinical Research Network program. J Clin Invest. 2013;3(11):1015-21. 